Case Report

\title{
Extraintestinal Salmonellosis in the Immunocompromised: An Unusual Case of Pyomyositis
}

\author{
Veeraraghavan Meyyur Aravamudan, ${ }^{1}$ Phang Kee Fong, ${ }^{1}$ Pavel Singh, ${ }^{2}$ \\ Jong Sze Chin, ${ }^{1}$ Yang Shiyao Sam, ${ }^{1}$ and P. A. Tambyah ${ }^{1,3}$ \\ ${ }^{1}$ Department of Medicine, National University Hospital, 5 Lower Kent Ridge Road, Singapore 119074 \\ ${ }^{2}$ Department of Diagnostic Imaging, National University Hospital, 5 Lower Kent Ridge Road, Singapore 119074 \\ ${ }^{3}$ Division of Infectious Diseases, National University Hospital, Singapore \\ Correspondence should be addressed to Veeraraghavan Meyyur Aravamudan; veera_meyyur_aravamudan@nuhs.edu.sg
}

Received 12 June 2017; Accepted 23 August 2017; Published 11 September 2017

Academic Editor: Ting Fan Leung

Copyright (c) 2017 Veeraraghavan Meyyur Aravamudan et al. This is an open access article distributed under the Creative Commons Attribution License, which permits unrestricted use, distribution, and reproduction in any medium, provided the original work is properly cited.

\begin{abstract}
Salmonella infection can cause a wide range of presentations, predominantly gastrointestinal but occasionally with cardiovascular or other extraintestinal manifestations. The diagnosis of extraintestinal salmonellosis requires a high degree of clinical suspicion and should be considered in patients with deep-seated abscesses especially if they are immunocompromised. We present a case of salmonella causing gastroenteritis complicated by an intramuscular abscess of the left leg. With prompt recognition and multidisciplinary management, the patient recovered with no serious sequela.
\end{abstract}

\section{Introduction}

Salmonella infection can cause a wide range of presentations especially in immunocompromised hosts. In this case, we describe a 55-year-old Chinese Singaporean man, who presented with acute onset of nonbilious and nonbloody vomiting with diarrhoea and left leg pain. He was found to have gastroenteritis caused by Salmonella Group C complicated by an intramuscular abscess of his left leg. We reviewed the literature in the context of this unusual presentation.

\section{Case Report}

A 55-year-old Chinese Singaporean man with a background of Polycythemia Rubra Vera (PRV), currently on treatment with hydroxyurea and anagrelide, diabetes mellitus, and hypertension, was admitted with a four-day history of nonbilious, nonbloody vomiting accompanied with nonbloody diarrhea. This was associated with fever and also left leg pain and swelling. He had no other infective symptoms including cough, sputum production, or dysuria.
Physical examination revealed pyrexia (temperature: 38.9 degrees Celsius), blood pressure of $140 / 60 \mathrm{mmHg}$, and pulse rate of $80 / \mathrm{min}$. His left calf was erythematous, swollen, warm, and tender. His abdomen was soft and nontender with no bruits. No pitting oedema was present. Routine blood investigations showed normal inflammatory markers and biochemistry (Table 1). Ultrasound of the left lower limbs showed thrombosis of the left posterior tibial vein. Blood and stool cultures on admission grew Group C Salmonella.

He was started on intravenous ceftriaxone and subcutaneous low molecular weight heparin (LWMH) for deep venous thrombosis.

However, he had persistent fever and became hypotensive on the 10th hospital day. Repeat blood cultures grew Group C Salmonella, but repeat stool culture grew Group B Salmonella. Transthoracic echocardiogram showed no evidence of vegetation. Computed tomography (CT) aortography did not reveal aortitis.

Magnetic Resonance Imaging (MRI) of the left lower limb was performed. It showed a multiloculated collection with enhancing rim and septa centered within the tibialis posterior 
TABLE 1: Initial blood test results.

(a)

\begin{tabular}{|c|c|c|c|}
\hline Test & Results & Unit & Reference interval \\
\hline White blood cell & 7 & $\times 10^{9} / \mathrm{L}$ & $3.40-9.60$ \\
\hline Red blood cells & 3.06 & $\times 10^{12} / \mathrm{L}$ & $3.70-9.60$ \\
\hline Haemoglobin & 10.9 & $\mathrm{~g} / \mathrm{dL}$ & $10.9-15.1$ \\
\hline Mean cell volume & 105.6 & $\mathrm{fL}$ & $80.0-95.0$ \\
\hline Mean corpuscular haemoglobin & 35.6 & pg & $27.0-33.0$ \\
\hline Mean corpuscular haemoglobin Concentration & 33.7 & $\mathrm{~g} / \mathrm{dL}$ & $32.0-36.0$ \\
\hline Haematocrit & 32.3 & $\%$ & $32.7-44.4$ \\
\hline Platelets & 941 & $\times 10^{9} / \mathrm{L}$ & $132-372$ \\
\hline Mean platelet volume & 11.7 & $\mathrm{fL}$ & $8.7-12.2$ \\
\hline Red cell distribution width & 17.8 & $\%$ & $11.4-14.8$ \\
\hline Sodium & 135 & $\mathrm{mmol} / \mathrm{L}$ & $135-145$ \\
\hline Potassium & 4.0 & $\mathrm{mmol} / \mathrm{L}$ & $3.5-5.0$ \\
\hline Chloride & 99 & $\mathrm{mmol} / \mathrm{L}$ & $95-110$ \\
\hline Carbon dioxide & 23 & $\mathrm{mmol} / \mathrm{L}$ & $22-31$ \\
\hline Creatinine & 113 & umol/L & $50-90$ \\
\hline Urea & 7.3 & $\mathrm{mmol} / \mathrm{L}$ & $2.0-6.5$ \\
\hline Glucose & 8.6 & $\mathrm{mmol} / \mathrm{L}$ & $4.0-7.8$ \\
\hline Albumin & 38 & $\mathrm{~g} / \mathrm{L}$ & $38-48$ \\
\hline Bilirubin, total & 2 & $\mathrm{umol} / \mathrm{L}$ & $5-30$ \\
\hline Bilirubin, conjugated & 1 & umol/L & $0-5$ \\
\hline Aspartate aminotransferase & 40 & $\mathrm{U} / \mathrm{L}$ & $10-50$ \\
\hline Alanine aminotransferase & 35 & $\mathrm{U} / \mathrm{L}$ & $10-70$ \\
\hline Alkaline phosphatase & 99 & $\mathrm{U} / \mathrm{L}$ & $40-130$ \\
\hline Lactate dehydrogenase & 693 & $\mathrm{U} / \mathrm{L}$ & $250-580$ \\
\hline Calcium, total & 2.30 & $\mathrm{mmol} / \mathrm{L}$ & $2.15-2.55$ \\
\hline C-reactive protein & 125 & $\mathrm{mg} / \mathrm{L}$ & $0-10$ \\
\hline
\end{tabular}

(b)

\begin{tabular}{lccc}
\hline Blood culture & Drugs & Susceptibility & MIC \\
\hline & Ampicillin & Sensitive & $\leq 2 \mathrm{mg} / \mathrm{L}$ \\
Blood culture grew Salmonella Group C & Ceftriaxone & Sensitive & Resistant \\
& Ciprofloxacin & Sensitive & $0.500 \mathrm{mg} / \mathrm{L}$ \\
& Cotrimoxazole & & $40.00 \mathrm{mg} / \mathrm{L}$ \\
\hline
\end{tabular}

(c)

\begin{tabular}{lccc}
\hline Stool culture & Drugs & Susceptibility & MIC \\
\hline & Ampicillin & Sensitive & $\leq 2 \mathrm{mg} / \mathrm{L}$ \\
Stool culture grew Salmonella Group C & Ceftriaxone & Sensitive & \\
& Ciprofloxacin & Resistant & $0.380 \mathrm{mg} / \mathrm{L}$ \\
& Cotrimoxazole & Sensitive & $\leq 20 \mathrm{mg} / \mathrm{L}$ \\
\hline
\end{tabular}

(d)

\begin{tabular}{|c|c|c|c|}
\hline Stool culture & Drugs & Susceptibility & MIC \\
\hline \multirow{5}{*}{ Stool culture grew Salmonella Group B } & Ampicillin & Resistant & $\geq 32 \mathrm{mg} / \mathrm{L}$ \\
\hline & Ceftriaxone & Resistant & $>256.000$ \\
\hline & Ciprofloxacin & Sensitive & 0.008 \\
\hline & Cotrimoxazole & Sensitive & $\leq 20 \mathrm{mg} / \mathrm{L}$ \\
\hline & Azithromycin & sensitive & 4.000 \\
\hline
\end{tabular}




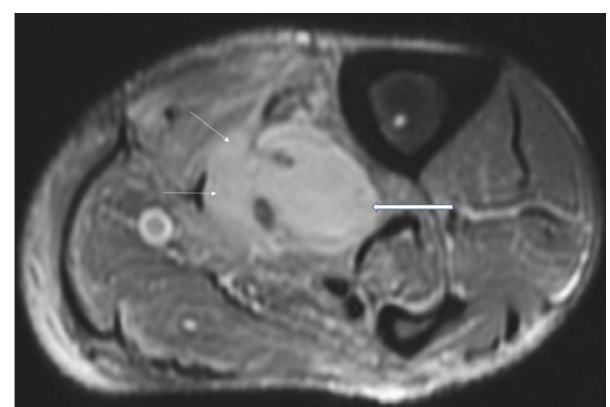

FIGURE 1: Axial, T2 weighted, fat saturated, STIR MR image showing loculated fluid signal within the tibialis posterior muscle (thick white arrows), suggestive of a collection. Also noted are diffuse T2 hyperintense signal changes in the surrounding musculature (thin white arrows), compatible with myositis.

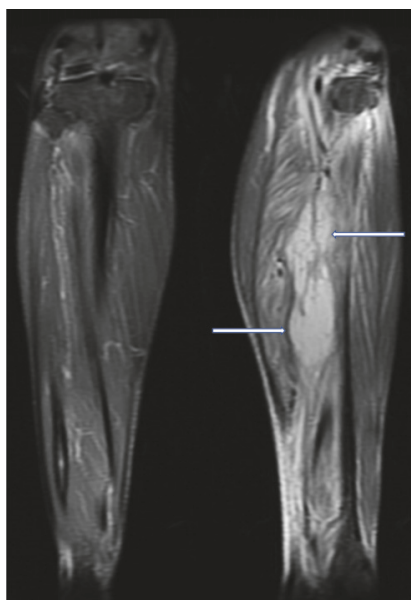

FIGURE 2: Coronal, T2 weighted, fat saturated, STIR MR image showing loculated fluid signal within the tibialis posterior muscle (thick white arrows), suggestive of a collection. Also noted are diffuse $\mathrm{T} 2$ hyperintense signal changes in the surrounding musculature (thin white arrows), compatible with myositis.

muscle, measuring approximately $13.9 \times 4.4 \times 4 \mathrm{~cm}$ with adjacent myositis and fasciitis (Figures 1, 2, 3, 4, and 5).

He was referred to orthopaedics for incision and drainage. Intraoperatively, a multiloculated intramuscular abscess in posterior tibialis muscle was observed; $15 \mathrm{mls}$ of frank pus was drained. Two drains were inserted, with the inferior drain at the residual space of the posterior tibialis muscle and superior drain into the space between the posterior tibialis and soleus. Intraoperative pus culture grew Group B Salmonella.

His condition improved dramatically following the drainage with resolution of pyrexia and hypotension. IV ceftriaxone was continued for a total duration of six weeks with oral metronidazole. LWMH was administered for six weeks as well. He remained well six months after discharge.

\section{Discussion}

Typhoid fever, caused by Salmonella enterica serovar Typhi, is an important disease in many developing countries. It is

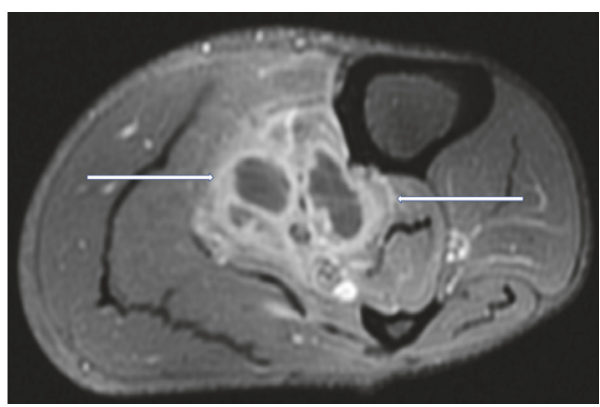

FIGURE 3: T2 weighted, fat saturated, postcontrast, axial MR image of left calf showing a multiloculated fluid collection with associated enhancing rim and septations (white arrows) within the tibialis posterior muscle, compatible with an intramuscular abscess.

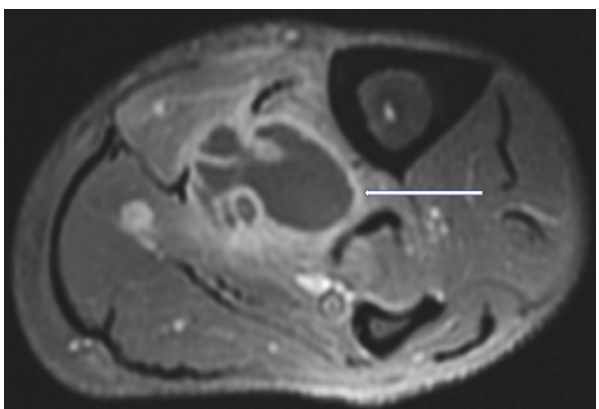

FIGURE 4: T2 weighted, fat saturated, postcontrast, axial MR image of left calf at another location, again showing a multiloculated fluid collection with associated enhancing rim and septations (white arrows) within the tibialis posterior muscle, compatible with an intramuscular abscess.

estimated that there are approximately 22 million typhoid cases and 200,000 deaths per year worldwide [1].

Enteric fever is caused by Salmonella Typhi or Paratyphi. In contrast, focal infections such as osteomyelitis and endovascular infections tend to be caused by nontyphoidal salmonellae (NTS). While NTS commonly cause endovascular infections with mycotic aneurysm of aorta as the most common site $[2,3]$, myositis and other soft tissue infections have been previously described $[4,5]$. The type of infection depends on both host factors and, to a lesser degree, on the serotype of salmonellae [6].

Transmission usually occurs by the oral-faecal route [7] via consuming contaminated drinking water and food sources, such as poultry, eggs, dairy products, vegetables, and fruits. Our patient did not give a clear history of dietary indiscretion but half-boiled eggs are commonly consumed in Singapore and other parts of Southeast Asia [8,9].

In microbiology laboratories, salmonellae are now rapidly identified using automated systems [10]. Interestingly, two different salmonellae strains were found in our patient and this may reflect his underlying heavily immunocompromised state [11].

Salmonella infection of the thigh has previously been reported but in association with a pseudoaneurysm of the femoral artery [4]. 


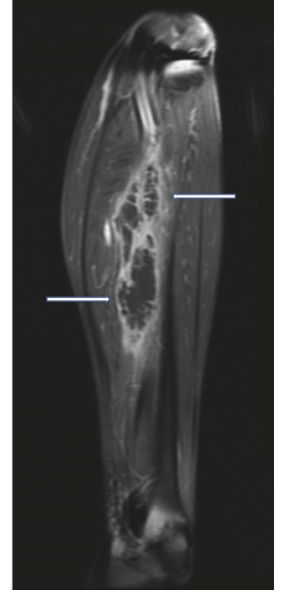

FIGURE 5: T2 weighted, fat saturated, postcontrast, coronal MR image of left calf showing a multiloculated fluid collection with associated enhancing rim and septations (white arrows) within the tibialis posterior muscle, compatible with an intramuscular abscess.

Current literature regarding epidemiology of extraintestinal salmonellosis in immunocompromised patients remains scarce. A Malaysian study on NTS bacteraemia found that 55 out of 56000 blood cultures collected over four years grew NTS. An extraintestinal focus was found in 30\% of cases, most commonly the lung and soft tissues (7.3\% each). The study also described higher mortality in immunocompromised patients $(30.6 \%$ versus $5.3 \%$ in immunocompetent patients) [12].

Atherosclerosis and HIV infections are the most common predisposing factors for systemic salmonella infections [13, $14]$, although recently the emergence of defects or autoantibodies directed against the interferon gamma pathway has been shown to be a major underlying cause of systemic salmonella infections, particularly in Southeast Asia [15].

Warning signs of extraintestinal infection would include localized tenderness in the musculoskeletal system, pleuritic pain suggesting a pleural pathology, chest pain, or embolic phenomena suggesting a mycotic aneurysm.

In our patient, despite adequate treatment of his salmonella bloodstream infection and his deep vein thrombosis with ceftriaxone and LWMH, he became hypotensive and it was only with drainage of his intramuscular abscess that his condition began to improve.

It is important that physicians be alert for timely diagnosis of extraintestinal salmonella infections, especially in immunocompromised patients with the risk of complications which may be potentially fatal [16].

\section{Learning Value}

The diagnosis of extraintestinal salmonellosis necessitates a high degree of clinical suspicion. The majority of individuals with immunocompromised immune systems present with extraintestinal infections. A multidisciplinary approach is needed to treat immunocompromised patients with extraintestinal salmonella infections and abscesses.

\section{Conflicts of Interest}

Dr. Veeraraghavan Meyyur Aravamudan, Dr. Phang Kee Fong, Dr. Jong Sze Chin, Dr. Yang Shiyao Sam, Dr. Pavel Singh, and Dr. P. A. Tambyah have no disclosures to make directly related to the study and no conflicts of interest and no funding was received for the preparation of this manuscript. Dr. Tambyah has received research support from GSK, Sanofi-Pasteur, and Janssen in the last six months.

\section{Authors' Contributions}

Veeraraghavan Meyyur Aravamudan, Phang Kee Fong, Jong Sze chin, and Yang Shiyao Sam helped in the compilation of the text and literature search. Pavel Singh helped in radiological images and literature search. P. A. Tambyah helped in the compilation of the text, literature search, and editing the manuscript.

\section{References}

[1] S. Kariuki, G. Revathi, J. Kiiru et al., "Typhoid in Kenya is associated with a dominant multidrug-resistant Salmonella enterica serovar Typhi haplotype that is also widespread in Southeast Asia," Journal of Clinical Microbiology, vol. 48, no. 6, pp. 2171-2176, 2010.

[2] A Clinical Audit of Presentation and Outcome of Salmonella Septicaemia Ann Acad Med Singapore, 33, 749-53, 2004.

[3] A. G. Habib, "Adjacent Organ Involvement in Salmonella Aortic Aneurysms," Scandinavian Journal of Infectious Diseases, vol. 35, no. 6-7, pp. 415-417, 2009.

[4] P. Björkman, A. Nilsson, and K. Riesbeck, "A pilot with pain in his leg: Thigh abscess caused by Salmonella enterica serotype Brandenburg," Journal of Clinical Microbiology, vol. 40, no. 9, pp. 3530-3531, 2002.

[5] K. Minami, M. Sakiyama, H. Suzuki, and N. Yoshikawa, "Pyomyositis of thevastusmedialis muscle associated with salmonella enteritidis in a child," Pediatric Radiology, vol. 33, no. 7, pp. 492-494, 2003.

[6] Patient Info, "Salmonella Gastroenteritis," http://patient.info/ doctor/salmonella-gastroenteritis.

[7] N. Aliya and P. K. Ram, "Burden of typhoid and paratyphoid fever in a densely populated urban community," International Journal of Infectious Diseases, vol. 14, pp. e99-e103, 2010.

[8] O. Putcharoen and C. Suankratay, "Salmonella gas-forming femoral osteomyelitis andpyomyositis: the first case and review of the literature," Journal of the Medical Association of Thailand, vol. 90, no. 9, p. 1943, 2007.

[9] J. Collazos, J. Mayo, E. Martinez, and M. S. Blanco, "Muscle infections caused by salmonella species: case report and review," Clinical Infectious Diseases, vol. 29, no. 3, pp. 673-677, 1999.

[10] O. Gal-Mor, E. C. Boyle, and G. A. Grassl, "Same species, different diseases: how and why typhoidal and non-typhoidal salmonella entericaserovars differ," Front Microbiol, vol. 4, pp. 391-10, 2014.

[11] K. S. Kohl, S. M. Marcy, M. Blum et al., "Fever after immunization: current concepts and improved future scientific understanding," Clinical Infectious Diseases, vol. 39, no. 3, pp. 389-394, 2004.

[12] A. Dhanoa and Q. K. Fatt, "Non-typhoidal Salmonella bacteraemia: Epidemiology, clinical characteristics and its' association 
with severe immunosuppression," Annals of Clinical Microbiology and Antimicrobials, vol. 8, article no. 15, 2009.

[13] A. Dhanoa and Q. K. Fatt, "Non-typhoidal salmonella bacteraemia: epidemiology, clinical characteristics and its' association with severe immunosuppression," Annals of Clinical Microbiology and Antimicrobials, vol. 8, p. 15, 2009.

[14] J. Kedzierska, B. Piatkowska-Jakubas, and A. Kedzierska, "Clinical presentation ofextraintestinal infections caused by nontyphoid salmonella serotypes among patients at the university hospital in cracow during a 7-year period," Polish Journal of Microbiology, vol. 57, no. 1, pp. 41-47, 2008.

[15] E. H. Tham, C. Huang, J. Y. Soh et al., "Neutralizing antiinterferon-gamma autoantibody levels may not correlate with clinical course of disease," Clinical Infectious Diseases, vol. 63, no. 4, pp. 572-573, 2016.

[16] J. H. Hwang, G. W. Shin, and C. S. Lee, "Community-Onset Pyomyositis Caused By A Salmonella EntericaSerovarEnteritidis Sequence Type1l Strain Producing CTX-M-15 ExtendedSpectrum ?-Lactamase," J ClinMicrobiol, vol. 53, no. 4, pp. 143941, 2015. 


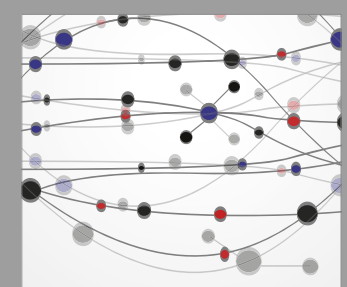

The Scientific World Journal
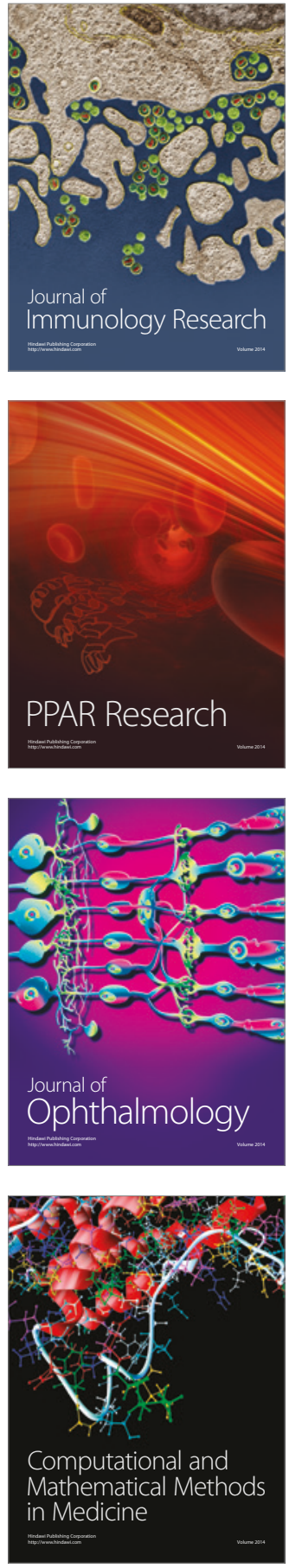

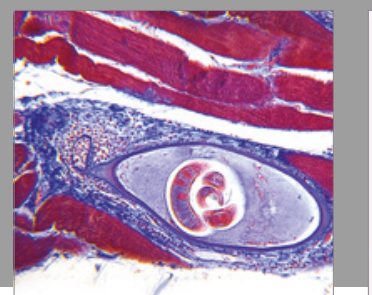

Gastroenterology Research and Practice
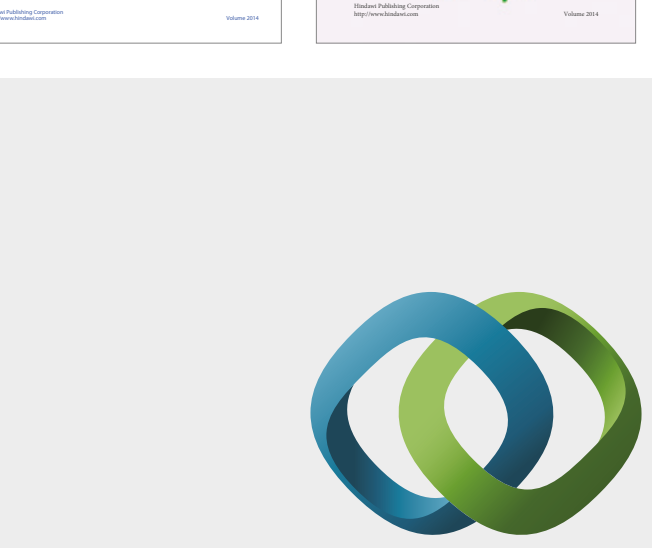

\section{Hindawi}

Submit your manuscripts at

https://www.hindawi.com
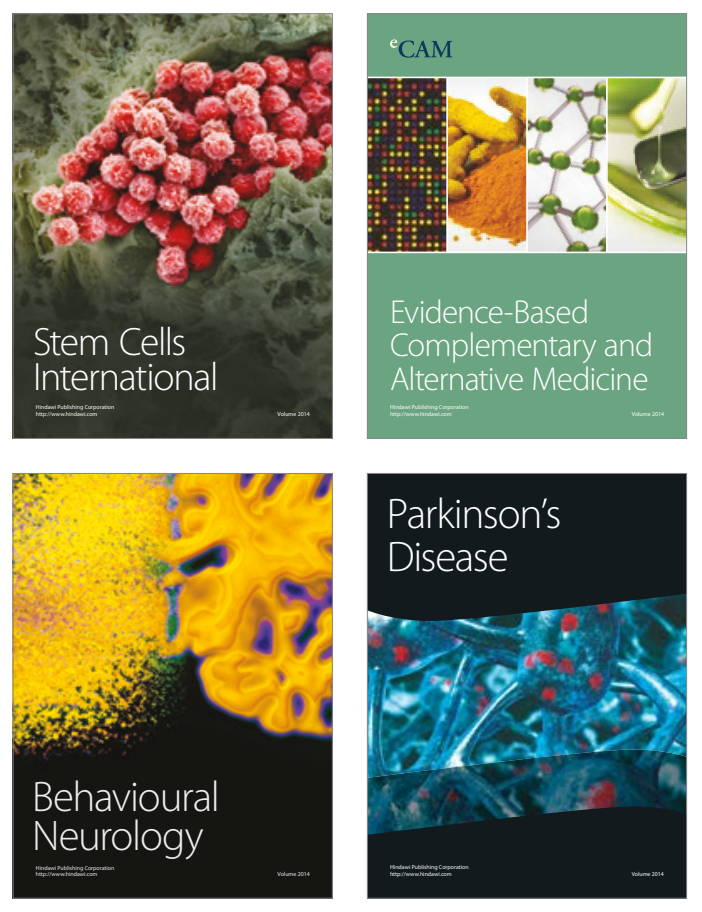
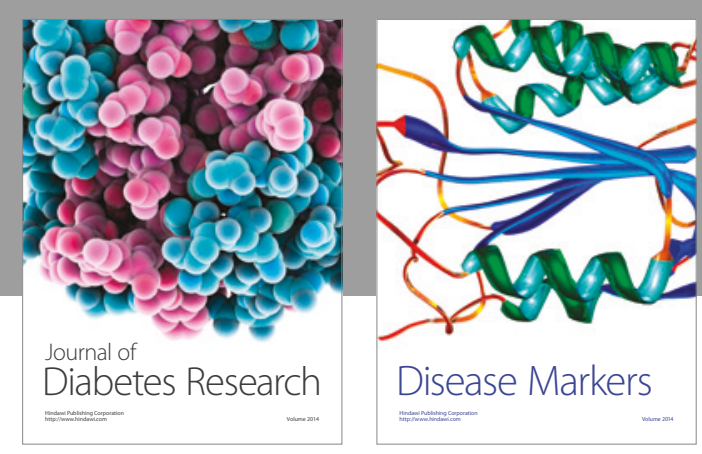

Disease Markers
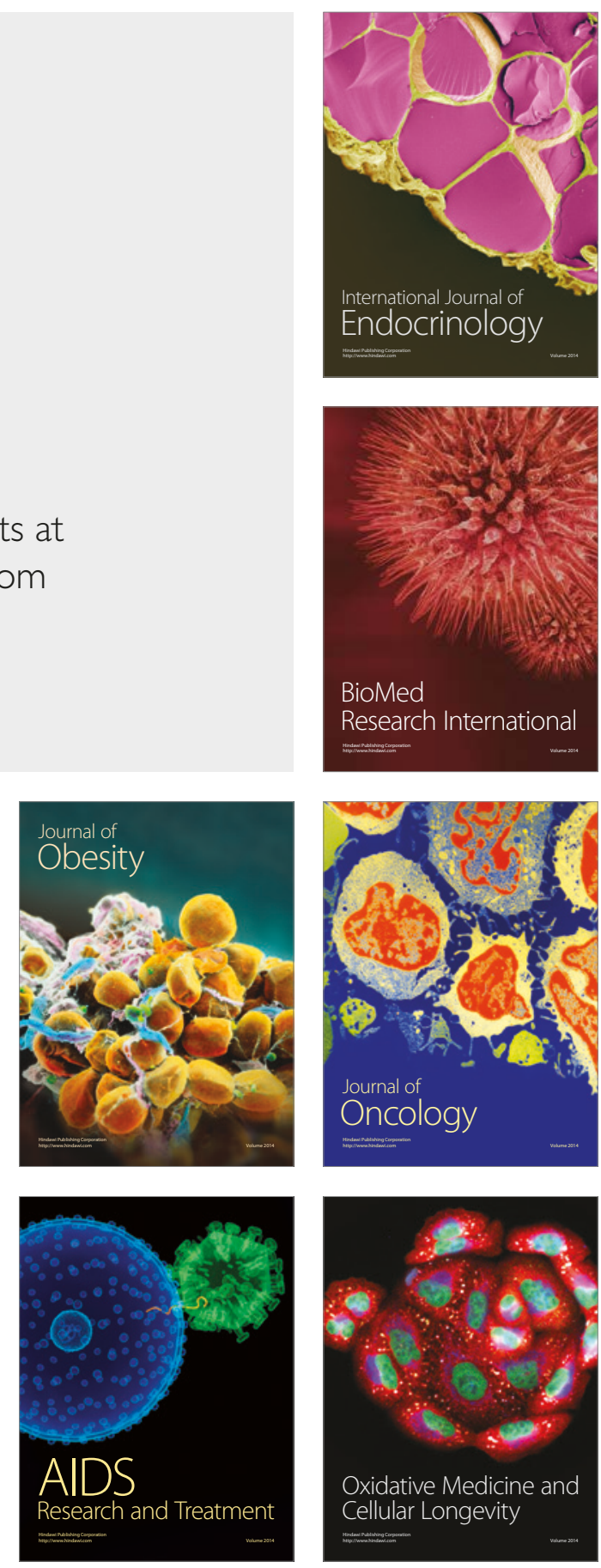